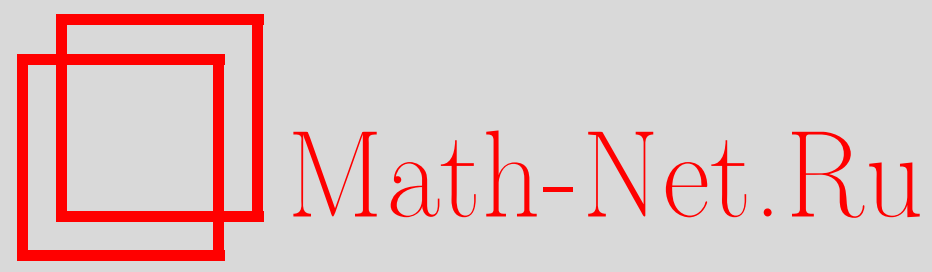

М. Г. Плотников, Вопросы единственности для некоторых классов рядов Хаара, Матем. заметки, 2004, том 75, выпуск 3, 392-404

DOI: https://doi.org/10.4213/mzm43

Использование Общероссийского математического портала Math-Net.Ru подразумевает, что вы прочитали и согласны с пользовательским соглашением http://www . mathnet.ru/rus/agreement

Параметры загрузки:

IP : 54.224 .60 .19

26 апреля 2023 г., 13:50:16 


\section{ВОПРОСЫ ЕДИНСТВЕННОСТИ ДЛЯ НЕКОТОРЫХ КЛАССОВ РЯДОВ ХААРА}

\section{М.Г. Плотников}

Работа посвящена изучению множеств относительной единственности для рядов Хаара. Изучается целый класс условий на поведение ряда Хаара, включая условие Арутюняна-Талаляна. В работе введены новые числовые характеристики совершенных множеств. С их помошью получены необходимые, а также достаточные условия того, что данное множество является множеством относительой единственности при заданных условиях. Тем самым обобщаются результаты Г. М. Мушегяна, полученные в 1967 г. Также показывается наличие при $0<p<2$ совершенных $\mathrm{U}$-множеств с $\mathrm{G}(p)$-условиями, введенньми В. Уэйдом в 1981 г. и дается способ построения таких множеств.

Библиограффия: 7 названий.

В статье будут рассмотрены некоторые вопросы единственности для рядов Хаара. Мы используем следующее определение функиий Хаара: $\chi_{1}(x) \equiv 1$ для $x \in[0,1]$; если $n=2^{k}+i$, где $k \geqslant 0,1 \leqslant i \leqslant 2^{k}$, то

$$
\chi_{n}(x)= \begin{cases}2^{k / 2} & \text { при } x \in\left(\frac{2 i-2}{2^{k+1}}, \frac{2 i-1}{2^{k+1}}\right), \\ -2^{k / 2} & \text { при } x \in\left(\frac{2 i-1}{2^{k+1}}, \frac{2 i}{2^{k+1}}\right), \\ 0 & \text { при }\left[\frac{2 i-2}{2^{k+1}}, \frac{2 i}{2^{k+1}}\right] .\end{cases}
$$

В точках 0 и 1 функцию $\chi_{n}(x)$ мы полагаем равной пределу справа (слева) функции $\chi_{n}(x)$, а в остальных точках отрезка $[0,1]$ функция $\chi_{n}(x)$ равна среднему арифметическому правого и левого пределов.

Известно, что пустое множество является U-множеством для рядов Хаара (см. [1][3]). Это означает, что если ряд Хаара сходится к нулю вне пустого множества, т.е. всюду на $[0,1]$, то все его коэффициенты нулевые. В то же время легко построить ряд Хаара (см. [4]), у которого не все коэффициенты нулевые, сходящийся к нулю всюду на $[0,1]$, кроме заданной точки $x$. Это означает, что любое одноточечное множество $A \subset[0,1]$ есть М-множество для рядов Хаара. В связи с этим естественно рассматривать множества относительной единственности для рядов Хаара, наложив ограничения на поведение коэффициентов (или частичных сумм) ряда.

Работа выполнена при поддержке Российского фонда фундаментальных исследований, грант № 02-01-00428. 
Будем пользоваться следующей терминологией. Рассматривая класс рядов Хаара

$$
\sum_{n=1}^{\infty} a_{n} \chi_{n}(x)
$$

удовлетворяющих некоторому условию (а); скажем, что множество $A$ является М-множеством для рядов Хаара с условием (a), если существует ряд (1), удовлетворяющий условию (а), которьй сходится к нулю вне $A$ и не все коэффициенты которого нулевые. Если такого ряда не существует, т.е. из сходимости к нулю ряда (1) с условием (a) вне множества $A$ следует, что $a_{n}=0$ для всех $n=1,2, \ldots$, то множество $A$ назовем $\mathrm{U}$-множеством для рядов (1) с условием (а). Иначе такие множества назьвают множествами относительной единственности.

В 1967 году Г. М. Мушегян доказал (см. [5, теорема 1]), что если рассматривать ряды Хаара с условием Арутюняна-Талаляна

$$
\frac{a_{n_{k}}}{\chi_{n_{k}}(x)} \rightarrow 0
$$

где $n_{k}$ - последовательность номеров таких, что $\chi_{n_{k}}(x) \neq 0$, то множество $A$ является М-множеством для ряда (1) с условием (2) тогда и только тогда, когда $A$ содержит совершенное подмножество. Отсюда получается, что борелевское множество является $\mathrm{U}$-множеством для рядов (1) с условием (2), только если оно не более чем счетно. В нашей работе мы наложим более сильные ограничения на поведение ряда Хаара и получим болееширокий класс U-множеств. Задав $\alpha \in[0,1)$, рассмотрим класс рядов Хаара, удовлетворяющих условию

$$
S_{N}(x)=o\left(N^{1-\alpha}\right),
$$

где $S_{N}(x)=\sum_{n=1}^{N} a_{n} \chi_{n}(x)$, а "o" - "свое" для каждого $x$.

Дадим ряд определений (см. [3], [6]). Интервал вида $\left[p / 2^{n},(p+1) / 2^{n}\right]$, где $n=$ $0,1, \ldots, p=0, \ldots, 2^{n}-1$, назьвается двоичным интервалом, а число $n$ - его рангом. Точка отрезка $[0,1]$, имеющая вид $p / 2^{n}$, назьвается двоично-рачиональной (обозначение $x \in \mathbb{R})$, а если точка отрезка $[0,1]$ не имеет вид $p / 2^{n}$, то она называется двоично-иррачиональной (обозначение $x \in \mathbb{I}$ ). Последовательность двоичных интервалов $\left\{\Delta_{n}\right\}$ назьвается основной для точки $x$, если $x \in \Delta_{n}$, ранг $\Delta_{n}$ равен $n$ и $\Delta_{n} \subset \Delta_{n-1}$. Если $x \in \mathbb{I}$, то для точки $x$ существует одна, а если $x \in \mathbb{R}$ и $x \neq 0,1$, то две основные последовательности.

Пусть задан ряд (1). Поставим ему в соответствие функцию двоичного интервала

$$
\Psi(\Delta)=\sum_{m=1}^{\infty} \int_{\Delta} a_{m} \chi_{m}(x) d x .
$$

В работе [6] (см. теорема 1) показано, что если $\Delta_{n}-$ двоичньй интервал ранга $n$, то сумма в правой части конечна и верно равенство

$$
S_{2^{n}}(x)=\frac{\Psi\left(\Delta_{n}\right)}{\left|\Delta_{n}\right|},
$$

где $x$ - любая внутренняя точка $\Delta_{n}$. В силу конечной аддитивности интеграла Римана функция $\Psi(\Delta)$ - аддитивная функция двоичного интервала. Покажем, что соответствие 
между рядами (1) и аддитивньми функциями двоичного интервала на самом деле взаимно-однозначно и для любой аддитивной функции двоичного интервала $\Psi(\Delta)$ существует (притом единственный) ряд (1), для которого $\Psi(\Delta)$ определяется по формуле (4).

Обозначим через $S_{n}^{i}$, где $n=0,1, \ldots, i=1, \ldots, 2^{n}$, постоянное значение частичной суммы $S_{2^{n}}(x)$ на открытом двоичном интервале $\Delta_{n}^{i}=\left((i-1) / 2^{n}, i / 2^{n}\right)$. Нетрудно заметить, что набор чисел $S_{n}^{i}$ удовлетворяет свойству

$$
2 S_{n}^{i}=S_{n+1}^{2 i-1}+S_{n+1}^{2 i}
$$

Покажем, что на самом деле существует взаимно-однозначное соответствие между тремя объектами: рядами Хаара, аддитивными функциями двоичного интервала и наборами чисел $S_{n}^{i}$, где $n=0,1, \ldots, i=1, \ldots, 2^{n}$, удовлетворяющих свойству (6), причем при данном соответствии эти три объекта будут связаны равенствами (4) и (5), а также равенством

$$
S_{2^{n}}(x)=S_{n}^{i}
$$

(если $x$ - внутренняя точка $\Delta_{n}^{i}$ ). Действительно, между аддитивными функциями двоичного интервала $\Psi(\Delta)$ и между наборами чисел $S_{n}^{i}$ можно осуществить взаимно-однозначное соответствие по формуле (5) (при этом аддитивность функции $\Psi(\Delta)$ будет равносильна выполнению свойства (6) для набора $\left.S_{n}^{i}\right)$. Полагая $a_{1}=S_{0}^{1}$ и

$$
a_{n}=\frac{S_{k+1}^{2 i-1}-S_{k}^{i}}{2^{k / 2}}=\frac{S_{k+1}^{2 i}-S_{k}^{i}}{-2^{k / 2}}
$$

(последнее равенство вьполняется в силу условия (6)), если $n=2^{k}+i$, где $k \geqslant 0$, $1 \leqslant i \leqslant 2^{k}$, можно осуществить взаимно-однозначное соответствие между рядами Хаара и наборами чисел $S_{n}^{i}$, удовлетворяющих свойству (6). При этом соответствии в силу свойства (8) будет выполняться равенство (7). Таким образом, существование взаимно-однозначного соответствия между тремя объектами доказано. Остается только заметить, что наличие условий (6) и (7) при этом соответствии гарантирует то, что аддитивная функция двоичного интервала $\Psi(\Delta)$ строится по соответствуюшему ряду Хаара по формуле (4).

Имея взаимно-однозначное соответствие между рядами Хаара и аддитивными функциями двоичного интервала, можно в терминах функции $\Psi(\Delta)$ сформулировать условия сходимости рядов Хаара.

УТВЕРЖДЕНИЕ 1. Если $x \in \mathbb{I}$, или $x$ равно 0 или 1, mo $S_{N}(x) \rightarrow S$ тогда $u$ только тогда, когда $\Psi\left(\Delta_{n}\right) /\left|\Delta_{n}\right| \rightarrow S$, где $\left\{\Delta_{n}\right\}$ - основная для точки $x$ последовательность.

ДокАЗАТЕЛьСТво см. в работе [6] (теорема 1).

УТВЕРЖДЕНИЕ 2. Если $x \in \mathbb{R} u x$ не равно 0 или 1, mo $S_{N}(x) \rightarrow S$ тогда $u$ только тогда, когда

$u$

$$
\frac{\Psi\left(\Delta_{n}^{1}\right)+\Psi\left(\Delta_{n}^{2}\right)}{2\left|\Delta_{n}^{1}\right|} \rightarrow S
$$

$$
\frac{\Psi\left(\Delta_{n}^{i}\right)-\Psi\left(\Delta_{n-1}^{i}\right) / 2}{\left|\Delta_{n}^{i}\right|} \rightarrow 0
$$

при $n \rightarrow \infty$, әде $\left\{\Delta_{n}^{i}\right\}-i$-я основная последовательность для точки $x, i=1,2$. 
ДокАЗАТЕЛЬСТво. Если $x=p / 2^{n}$, то $S_{N}(x) \rightarrow S$ тогда и только тогда, когда $S_{2^{N}}(x) \rightarrow S, S_{2^{N}+\frac{p}{2^{n}} 2^{N}}(x) \rightarrow S, S_{2^{N}+\frac{p}{2^{n}} 2^{N}+1}(x) \rightarrow S$ при $N \rightarrow \infty$. Это Эквивалентно тому что $S_{2^{N}}(x) \rightarrow S, S_{2^{N}+\frac{p}{2^{n}} 2^{N}}(x)-S_{2^{N}}(x) \rightarrow 0$ и $S_{2^{N}+\frac{p}{2^{n}} 2^{N}+1}(x)-$ $S_{2^{N}+\frac{p}{2^{n}} 2^{N}}(x) \rightarrow 0$ при $N \rightarrow \infty$. Рассмотрим условие $S_{2^{N}}(x) \rightarrow S$. При достаточно малом (зависящем от $N) \varepsilon$

$$
\begin{aligned}
S_{2^{N}}(x) & =\frac{1}{2}\left(S_{2^{N}}(x+\varepsilon)+S_{2^{N}}(x-\varepsilon)\right) \\
& =\frac{1}{2}\left(\frac{\Psi\left(\Delta_{N}^{1}\right)}{\left|\Delta_{N}^{1}\right|}+\frac{\Psi\left(\Delta_{N}^{2}\right)}{\left|\Delta_{N}^{2}\right|}\right)=\frac{\Psi\left(\Delta_{N}^{1}\right)+\Psi\left(\Delta_{N}^{2}\right)}{2\left|\Delta_{N}^{1}\right|}
\end{aligned}
$$

и условие $S_{2^{N}}(x) \rightarrow S$ равносильно условию (9). Покажем, что условие

$$
S_{2^{N}+\frac{p}{2^{n}} 2^{N}+1}(x)-S_{2^{N}+\frac{p}{2^{n}} 2^{N}}(x) \rightarrow 0
$$

равносильно условию (10) для правой основной последовательности для точки $x$ (и, аналогично, условие $S_{2^{N}}+\frac{p}{2^{n}} 2^{N}(x)-S_{2^{N}}(x) \rightarrow 0$ равносильно условию $(10)$, если $\left\{\Delta_{n}^{i}\right\}-$ левая основная последовательность для точки $x$ ). Если $i=2^{N}+p 2^{N} / 2^{n}$, то при достаточно малом $\varepsilon$

$$
\begin{aligned}
S_{i+1}(x)-S_{i}(x) & =a_{i+1} \chi_{i+1}(x)=\frac{1}{2} a_{i+1} \chi_{i+1}(x+\varepsilon) \\
& =\frac{1}{2}\left(S_{2^{N+1}}(x+\varepsilon)-S_{2^{N}}(x+\varepsilon)\right)=\frac{1}{2}\left(\frac{\Psi\left(\Delta_{N+1}\right)}{\left|\Delta_{N+1}\right|}-\frac{\Psi\left(\Delta_{N}\right)}{\left|\Delta_{N}\right|}\right) \\
& =\frac{1}{2}\left(\frac{\Psi\left(\Delta_{N+1}\right)}{\left|\Delta_{N+1}\right|}-\frac{\Psi\left(\Delta_{N}\right)}{2\left|\Delta_{N+1}\right|}\right)=\frac{1}{2} \frac{\Psi\left(\Delta_{N+1}\right)-\frac{1}{2} \Psi\left(\Delta_{N}\right)}{\left|\Delta_{N+1}\right|},
\end{aligned}
$$

что и доказывает равносильность условия $S_{2^{N}+\frac{p}{2^{n}} 2^{N}+1}(x)-S_{2^{N}}+\frac{p}{2^{n}} 2^{N}(x) \rightarrow 0$ и условия (10). Утверждение доказано.

Аналогично устанавливаются следующие утверждения.

УТВЕРЖДЕНИЕ 3. Если $x \in \mathbb{I}$, или $x$ равно 0 или 1, mo $S_{N}(x)=o\left(N^{1-\alpha}\right)$ mогда и только тогда, когда $\Psi\left(\Delta_{n}\right) /\left|\Delta_{n}\right|^{\alpha} \rightarrow 0$, где $\left\{\Delta_{n}\right\}-$ основная для точки $x$ последовательность.

УТВЕРЖДЕНИЕ 4. Если $x \in \mathbb{R} u x$ не равно 0 или 1, mo $S_{N}(x)=o\left(N^{1-\alpha}\right)$ mогда и только тогда, когда

$$
\frac{\Psi\left(\Delta_{n}^{1}\right)+\Psi\left(\Delta_{n}^{2}\right)}{\left|\Delta_{n}^{1}\right|^{\alpha}} \rightarrow 0 \quad u \quad \frac{\Psi\left(\Delta_{n}^{i}\right)-\frac{1}{2} \Psi\left(\Delta_{n-1}^{i}\right)}{\left|\Delta_{n}^{i}\right|^{\alpha}} \rightarrow 0
$$

при $n \rightarrow \infty$, әде $\left\{\Delta_{n}^{i}\right\}-i$-я основная последовательность для точки $x, i=1,2$.

Для нахождения U-множеств и М-множеств для рядов Хаара с условием (3) нам потребуются новые числовые характеристики совершенных множеств. Пусть $A \subset[0,1]-$ непустое совершенное множество. Построим по этому множеству аддитивную функцию двоичного интервала $\beta_{A}(\Delta)$. Положим $\beta_{A}([0,1])=1$. Условимся, что $\beta_{A}(\Delta)=0$ тогда и только тогда, когда $A \cap \Delta$ содержит не более чем конечное число точек. Далее, предположив, что функция $\beta_{A}$ задана на двоичном интервале $\Delta_{n}$ ранга $n$, определим 
$\beta_{A}$ на каждом из двух двоичных интервалов $\Delta_{n+1}^{1}$ и $\Delta_{n+1}^{2}$ ранга $n+1$, для которых $\Delta_{n}=\bigcup_{i=1}^{2} \Delta_{n+1}^{i}$. Если $\Delta_{n+1}^{i} \cap A$ содержит бесконечное число точек при $i=1,2$, то положим $\beta_{A}\left(\Delta_{n+1}^{i}\right)=\beta_{A}\left(\Delta_{n}\right) / 2$. Если $\Delta_{n+1}^{i} \cap A$ содержит бесконечное число точек ровно при одном значении $i \quad(i=1$ или $i=2)$, то для этого номера $i$ положим $\beta_{A}\left(\Delta_{n+1}^{i}\right)=\beta_{A}\left(\Delta_{n}\right)$, а для другого номера $\bar{i}$ положим $\beta_{A}\left(\Delta_{n+1}^{\bar{i}}\right)=0$. Если же $\Delta_{n+1}^{1} \cap A$ и $\Delta_{n+1}^{i} \cap A$ содержат лишь конечное число точек, то $\beta_{A}\left(\Delta_{n+1}^{i}\right)=0$ при $i=1,2$.

Теперь дадим ряд определений.

ОПРеДЕЛЕНИЕ 1. Пусть $A$ - непустое совершенное множество из [0,1]. Назовем $u н-$ дексом точки $x \in A$ относительно множества $A$ число ind ${ }_{A} x$ (или, возможно, два числа ind ${ }_{A}^{1} x$ и ind ${ }_{A}^{2} x$, если $\left.x \in \mathbb{R}\right), \operatorname{paвное~} \sup \left\{\alpha \geqslant 0: \beta_{A}\left(\Delta_{n}\right)=o\left(\left|\Delta_{n}\right|^{\alpha}\right)\right\}$, где $\left\{\Delta_{n}\right\}-$ основная последовательность для точки $x$, причем каждый двоичньй интервал $\Delta_{n}$ содержит бесконечное число точек из $A$.

Отметим, что данное определение корректно, так как в силу совершенности множества $A$ точка $x$ не является изолированной точкой множества $A$ и поэтому $\beta_{A}\left(\Delta_{n}\right)=o(1)$ $=o\left(\left|\Delta_{n}\right|^{0}\right)$ для основной для точки $x \in \mathbb{I}$ последовательности и хотя бы для одной из двух основных для точки $x \in \mathbb{R}$ последовательностей. Заметим, что $0 \leqslant \operatorname{ind}_{A} x \leqslant 1$. В случае $x \in A \cap \mathbb{R}$ точка $x$ имеет один индекс, если только одна из двух основных для точки $x$ последовательностей $\left\{\Delta_{n}^{i}\right\}$ такова, что $\Delta_{n}^{i} \cap A$ содержит бесконечное число точек, а если таких последовательностей две, то точка $x$ имеет два индекса ind ${ }_{A}^{1} x$ и ind ${ }_{A}^{2} x$.

ОПРЕДЕЛЕНИЕ 2. Назовем верхним индексом непустого совершенного множества $A \subset[0,1]$ число ind $A=\sup _{x \in A}$ ind $_{A} x$ и нижним индексом множества $A$ число ind $A$ $=\inf _{x \in A} \operatorname{ind}_{A} x$. Если верхний и нижний индексы множества $A$ совпадают, то их общее значение назовем индексом множсества $A$ (обозначение ind $A$ ).

Перейдем к формулировке и доказательству основных результатов.

Теорема 1. Пусть $A$ - М-мнохество для рядов (1) с условием (3). Тогда $A$ содержит совериенное подмножество $B$ с $\overline{\text { ind }} B \geqslant \alpha$.

ДокАЗАТЕЛЬСтво. Пусть $A$ - М-множество для рядов (1) с условием (3). Это означает, что существует ряд вида (1), удовлетворяющий условию (3), не все коэффициенты которого нулевые и $S_{N}(x) \rightarrow 0$ вне $A$. Построим по ряду $\sum_{n=1}^{\infty} a_{n} \chi_{n}(x)$ в соответствии с (4) аддитивную функцию двоичного интервала $\Psi(\Delta)$. Тогда сушествует такой двоичный интервал $\Delta_{0}$, что $\Psi\left(\Delta_{0}\right) \neq 0$. Без ограничения общности будем считать, что $\Psi\left(\Delta_{0}\right)>0$. Рассмотрим аддитивную функцию двоичного интервала $\Psi_{1}(\Delta)$ $=\Psi(\Delta)-\varepsilon|\Delta|$, где $\varepsilon$ настолько мало, что $\Psi_{1}\left(\Delta_{0}\right)>0$. Пусть $\left[(2 j+i) / 2^{n},(2 j+i+1) / 2^{n}\right]-$ двоичньй интервал, где $i$ равно 0 или 1 . Назовем число $i$ типом данного двоичного интервала. Пусть $\left\{\Delta_{n}\right\}$ - вложенная последовательность двоичных интервалов, причем ранг $\Delta_{n}$ равен $n$. Тогда, если среди типов $\Delta_{n}$ каждое из чисел 0 и 1 встречается бесконечное число раз, то $\left\{\Delta_{n}\right\}$ стягивается к точке $x \in \mathbb{I}$, а если одно из чисел 0 или 1 встречается среди типов $\Delta_{n}$ конечное число раз, то $\left\{\Delta_{n}\right\}$ стягивается к точке $x \in \mathbb{R}$. Этим мы будем пользоваться.

Докажем следующее вспомогательное утверждение.

Лемма. Пусть двоичный интервал $\Delta_{0}$ таков, что $\Psi_{1}\left(\Delta_{0}\right)>0$. Тогда можно построить набор двоичных интервалов $I_{l}^{k}, J_{l}^{k}$, где $l=0,1,2, \ldots, k=1, \ldots, 2^{l}(k u$ l не связаны с рангом), со следующими свойствами:

1) $I_{0}^{1}=J_{0}^{1}=\Delta_{0}$ 
2) $J_{l}^{k} \supseteq I_{l+1}^{2 k-1} \supseteq J_{l+1}^{2 k-1}, J_{l}^{k} \supseteq I_{l+1}^{2 k} \supseteq J_{l+1}^{2 k}$;

3) $\Psi_{1}\left(I_{l}^{k}\right)>0 u \Psi_{1}\left(J_{l}^{k}\right)>0$ nрu всех $k u l$;

4) тuп $I_{l}^{k}$ не совпадает с типом $J_{l}^{k}$ при всех $l \geqslant 1 u k=1, \ldots, 2^{l}$.

ДокАЗАТЕЛЬСтво лЕммЫ. Построим наш набор индукцией по $l$. При $l=0$ у нас есть $I_{0}^{1}=J_{0}^{1}=\Delta_{0}$. Пусть построены $I_{l}^{k}, J_{l}^{k}$ при $l=0, \ldots, n, k=1, \ldots, 2^{l}$ с заданными свойствами. Построим $I_{l+1}^{k}, J_{l+1}^{k}$ при всех $k=1, \ldots, 2^{l+1}$.

Из условия (3) следует, что $S_{N}(x)=o(N)$, а значит общий член ряда (1) есть $o(N)$. Пусть $x \in[0,1]$ и $\left\{\Delta_{n}\right\}$ - одна из основных для точки $x$ последовательностей. Пусть последовательность номеров $n_{s}$ такова, что $\Delta_{s-1}$ является носителем функции $\chi_{n_{s}}$. Тогда

$$
\begin{aligned}
a_{n_{s}} \chi_{n_{s}}(y) & =S_{2^{s}}(y)-S_{2^{s-1}}(y) \\
& =\frac{\Psi\left(\Delta_{s}\right)}{\left|\Delta_{s}\right|}-\frac{\Psi\left(\Delta_{s-1}\right)}{\left|\Delta_{s-1}\right|}=\frac{1}{\left|\Delta_{s}\right|}\left[\Psi\left(\Delta_{s}\right)-\frac{1}{2} \Psi\left(\Delta_{s-1}\right)\right]
\end{aligned}
$$

(здесь $y$ - любая внутренняя точка двоичного интервала $\Delta_{s}$ ). Раз это есть $o\left(n_{s}\right)$, то

$$
\Psi\left(\Delta_{s}\right)-\frac{1}{2} \Psi\left(\Delta_{s-1}\right)=\frac{1}{\left|\Delta_{s}\right|}\left[\Psi\left(\Delta_{s}\right)-\frac{1}{2} \Psi\left(\Delta_{s-1}\right)\right] \cdot \frac{1}{2^{s}}=\frac{1}{2^{s}} o\left(2^{s}\right)=o(1) .
$$

Таким образом, равенство $S_{N}(x)=o(N)$ влечет за собой условие

$$
\Psi\left(\Delta_{n+1}\right)-\frac{\Psi\left(\Delta_{n}\right)}{2} \rightarrow 0
$$

а если заметить, что $\Psi\left(\Delta_{n+1}\right)-\Psi\left(\Delta_{n}\right) / 2=\Psi_{1}\left(\Delta_{n+1}\right)-\Psi_{1}\left(\Delta_{n}\right) / 2 \rightarrow 0$, то из равенства $S_{N}(x)=o(N)$ следует выполнение условия

$$
\Psi_{1}\left(\Delta_{n+1}\right)-\frac{1}{2} \Psi_{1}\left(\Delta_{n}\right) \rightarrow 0
$$

для любой основной для точки $x$ последовательности $\left\{\Delta_{n}\right\}$.

Покажем, что если вьполняется условие (11), то невозможна ситуация, когда $\Psi_{1}\left(\Delta_{n}\right)$ $>0$, a $\Psi_{1}\left(\overline{\Delta_{n} \backslash \Delta_{n+1}}\right) \leqslant 0$ при всех $n \geqslant n_{0}$. Если это не так и такая ситуация возможна, то

$$
\begin{aligned}
\Psi_{1}\left(\Delta_{n+1}\right)-\frac{1}{2} \Psi_{1}\left(\Delta_{n}\right) & =\frac{1}{2}\left(\Psi_{1}\left(\Delta_{n+1}\right)-\Psi_{1}\left(\overline{\Delta_{n} \backslash \Delta_{n+1}}\right)\right) \\
& \geqslant \frac{1}{2} \Psi_{1}\left(\Delta_{n+1}\right)>0
\end{aligned}
$$

а значит, $\Psi_{1}\left(\Delta_{n+1}\right) \geqslant \Psi_{1}\left(\Delta_{n}\right)$, т.е. $\Psi_{1}\left(\Delta_{n}\right)$ не убывает. Получается, что

$$
\Psi_{1}\left(\Delta_{n}\right) \geqslant \Psi_{1}\left(\Delta_{n_{0}}\right)>0
$$

следовательно,

$$
\Psi_{1}\left(\Delta_{n+1}\right)-\frac{\Psi_{1}\left(\Delta_{n}\right)}{2} \geqslant \frac{\Psi_{1}\left(\Delta_{n}\right)}{2} \geqslant \frac{\Psi_{1}\left(\Delta_{n_{0}}\right)}{2}>0,
$$

что противоречит условию (11).

Раз ситуация, описанная в начале предыдущего абзаца, невозможна, то деля каждый раз двоичньй интервал пополам, мы найдем такой двоичньй интервал $M_{l}^{k} \subseteq J_{l}^{k}$, что 
$\Psi_{1}\left(M_{l}^{k}\right)>0$ и $\Psi_{1}\left(M_{k l}^{i}\right)>0$ при $i=1,2$, где $M_{k l}^{1}$ и $M_{k l}^{2}$ - неперекрывающиеся двоичные интервалы, составляющие в объединении $M_{l}^{k}$. Положим $I_{l+1}^{2 k-1}=M_{k l}^{1}, I_{l+1}^{2 k}=M_{k l}^{2}$. Теперь определим $J_{l+1}^{2 k-1}$ и $J_{l+1}^{2 k}$. Для этого найдем такие двоичные интервалы $P_{l}^{k} \subset$ $I_{l+1}^{2 k-1}, P_{k l}^{1}$ и $P_{k l}^{2}$, что $\Psi_{1}\left(P_{l}^{k}\right)>0, \Psi_{1}\left(P_{k l}^{i}\right)>0$ при $i=1,2$, и неперекрывающиеся двоичные интервалы $P_{k l}^{1}$ и $P_{k l}^{2}$ составляют в объединении $P_{l}^{k}$. Из двух интервалов $P_{k l}^{1}$ и $P_{k l}^{2}$ выберем тот интервал $P_{k l}^{j_{0}}$, тип которого не совпадает с типом $I_{l+1}^{2 k-1}$ и положим $J_{l+1}^{2 k-1}=P_{k l}^{j_{0}}$. Аналогично найдем такой интервал $J_{l+1}^{2 k} \subset I_{l+1}^{2 k}$, что $\Psi_{1}\left(J_{l+1}^{2 k}\right)>0$ и тип $J_{l+1}^{2 k}$ не совпадает с типом $I_{l+1}^{2 k}$. Лемма доказана.

Возобновим доказательство основной теоремы. В лемме мы построили набор двоичных интервалов со свойствами 1-4. Заметим, что множество

$$
L\left(\Delta_{0}\right)=\bigcap_{l=0}^{\infty} \bigcup_{k=1}^{2^{l}} I_{l}^{k}=\bigcap_{l=0}^{\infty} \bigcup_{k=1}^{2^{l}} J_{l}^{k}
$$

является совершенньм, лежит в $\Delta_{0}$, и в силу свойства 4 принадлежит $\mathbb{I}$. Так как $L\left(\Delta_{0}\right)$ $\subset I$, в случае $x \in L\left(\Delta_{0}\right)$ имеем

$$
\varlimsup_{n \rightarrow \infty} \frac{\Psi\left(\Delta_{n}\right)}{\left|\Delta_{n}\right|}=\varlimsup_{n \rightarrow \infty} \frac{\Psi_{1}\left(\Delta_{n}\right)}{\left|\Delta_{n}\right|}+\varepsilon \geqslant 0+\varepsilon=\varepsilon,
$$

т.е. в точках $L\left(\Delta_{0}\right)$ данный ряд не сходится к нулю, а значит, $L\left(\Delta_{0}\right) \subset A$. Таким образом, имея двоичньй интервал $\Delta_{0}$, на котором $\Psi\left(\Delta_{0}\right)>0$, можно найти совершенное множество $L\left(\Delta_{0}\right) \subset \Delta_{0}$, принадлежашее М-множеству $A$. Этим мы воспользуемся в дальнейшем.

Построим по индукции вложенную последовательность двоичных интервалов $\left\{\Delta_{n}\right\}_{n \geqslant n_{0}}$ (причем ранг $\Delta_{n}$ равен $n, \Psi_{1}\left(\Delta_{n}\right)>0, \Delta_{n_{0}}=\Delta_{0}$ ), а также числовую последовательность $\tau_{m_{k}}$. Сначала мы имеем интервал $\Delta_{n_{0}}=\Delta_{0}$ и пустой набор $\tau_{m_{k}}$. Пусть нами построен интервал $\Delta_{n}$ и набор (возможно, пустой) $\tau_{m_{1}}, \ldots, \tau_{m_{k_{0}}}$. Построим интервал $\Delta_{n+1}$. Для этого разобьем $\Delta_{n}$ на два неперекрьвающихся двоичных интервала $\Delta_{n+1}^{1}$ и $\Delta_{n+1}^{2}$. Так как по предположению $\Psi_{1}\left(\Delta_{n}\right)>0$, то либо ровно для одного номера $i_{0}$ имеем $\Psi_{1}\left(\Delta_{n+1}^{i_{0}}\right)>0$. В этом случае положим $\Delta_{n+1}=\Delta_{n+1}^{i_{0}}$ и заметим, что $\Psi_{1}\left(\Delta_{n+1}\right)=\Psi_{1}\left(\Delta_{n}\right)-\Psi_{1}\left(\Delta_{n+1}^{\overline{i_{0}}}\right) \geqslant \Psi_{1}\left(\Delta_{n}\right)$ (здесь $\overline{i_{0}}-$ номер $(1$ или 2$)$, не совпадающий c $\left.i_{0}\right)$. K набору $\tau_{m_{1}}, \ldots, \tau_{m_{k_{0}}}$ в этом случае ничего не добавляем. Либо для обоих номеров 1 и $2 \Psi_{1}\left(\Delta_{n+1}^{1}\right)>0$ и $\Psi_{1}\left(\Delta_{n+1}^{2}\right)>0$; тогда, используя аддитивность функции $\Psi_{1}$, найдем номер $i_{0}\left(i_{0}\right.$ равно 1 или 2$)$ и число $\tau_{m_{k_{0}+1}} \geqslant 0$ такие, что

$$
\Psi_{1}\left(\Delta_{n+1}^{i_{0}}\right)=\frac{\Psi_{1}\left(\Delta_{n}\right)}{2}\left(1+\tau_{m_{k_{0}+1}}\right), \quad \Psi_{1}\left(\Delta_{n+1}^{\overline{i_{0}}}\right)=\frac{\Psi_{1}\left(\Delta_{n}\right)}{2}\left(1-\tau_{m_{k_{0}+1}}\right),
$$

и положим $\Delta_{n+1}=\Delta_{n+1}^{i_{0}}$. $\mathrm{K}$ набору $\tau_{m_{1}}, \ldots, \tau_{m_{k_{0}}}$ в этом случае добавим число $\tau_{m_{k_{0}+1}}$, положив при этом $m_{k_{0}+1}=n+1$.

Заметим, что для выбранной последовательности $\left\{\Delta_{n}\right\}$ имеем неравенство

$$
\Psi_{1}\left(\Delta_{n}\right) \geqslant \Psi_{1}\left(\Delta_{n_{0}}\right) \cdot \prod_{m_{k} \leqslant n}\left(\frac{1+\tau_{m_{k}}}{2}\right)
$$


Возможны три варианта развития событий.

1. Последовательность $\left\{\Delta_{n}\right\}$ стягивается к точке $x \in \mathbb{I}$. Используя формулу (12), получим, что

$$
\begin{aligned}
\frac{\Psi\left(\Delta_{n}\right)}{\left|\Delta_{n}\right|} & =\frac{\Psi_{1}\left(\Delta_{n}\right)}{\left|\Delta_{n}\right|}+\varepsilon \geqslant \frac{\Psi_{1}\left(\Delta_{n_{0}}\right) \cdot \prod_{m_{k} \leqslant n}\left(\frac{1+\tau_{m_{k}}}{2}\right)}{\frac{1}{2^{n}}} \\
& \geqslant \frac{\Psi_{1}\left(\Delta_{n_{0}}\right) \cdot \frac{1}{2^{n-n_{0}}}}{\frac{1}{2^{n}}}=2^{n_{0}} \Psi_{1}\left(\Delta_{n_{0}}\right)>0 .
\end{aligned}
$$

Значит, в точке $x$ наш ряд не сходится к нулю и $x \in A$. Заметим, что последовательность $\tau_{m_{k}}$ будет бесконечной (фактически это доказано в начале теоремы). Возьмем интервалы $\Delta_{m_{k}}$, далее возьмем двоичные интервалы $\overline{\Delta_{m_{k}-1} \backslash \Delta_{m_{k}}}$ при $k=1,2, \ldots$, и процедурой, описанной в лемме, построим совершенные множества $L\left(\overline{\Delta_{m_{k}-1} \backslash \Delta_{m_{k}}}\right)$ $\subset A$. Это можно сделать, так как по построению не только $\Psi_{1}\left(\Delta_{m_{k}}\right)>0$, но и

$$
\Psi_{1}\left(\overline{\Delta_{m_{k}-1} \backslash \Delta_{m_{k}}}\right)>0 .
$$

Возьмем множество $B=\{x\} \cup \bigcup_{k=1}^{\infty} L\left(\overline{\Delta_{m_{k}-1} \backslash \Delta_{m_{k}}}\right)$. Покажем, что множество $B$ совершенно. Во-первых, $x$ не является изолированной точкой множества $B$, так как любая окрестность точки $x$ содержит все $\Delta_{m_{k}}$ для достаточно больших $k$, а значит, и бесконечное число точек из $B$. Любая другая точка множества $B$ не является изолированной из-за того, что она принадлежит некоторому совершенному множеству $\Delta_{m_{k}}$, полностью лежашему в $B$. Покажем, что множество $B$ замкнутое. Пусть $x_{1} \in \bar{B}$. Тогда существует последовательность $x_{N}, x_{N} \in B$ и $x_{N} \rightarrow x_{1}$. Если $x_{N}$ содержит точки из бесконечного числа подмножеств $\Delta_{m_{k}}$, то $x_{N} \rightarrow x$, а значит, $x_{1}=x \in B$. Если же $x_{N}$ содержит точки лишь из конечного числа подмножеств $\Delta_{m_{k}}$, то существует номер $k_{0}$ и последовательность $n_{l}$ такие, что $x_{n_{l}} \in \Delta_{m_{k_{0}}}$ и $x_{n_{l}} \rightarrow x_{1}$. В силу замкнутости множества $\Delta_{m_{k_{0}}}$ получим, что $x_{1} \in \Delta_{m_{k_{0}}} \subset B$. Итак, всегда $x_{1} \in B$. Замкнутость множества $B$ доказана. Таким образом, множество $B$ совершенно.

Покажем, что ind ${ }_{B} x \geqslant \alpha$, откуда сразу получим, что $\overline{\operatorname{ind}} B \geqslant \alpha$; а так как $B \subset A$, теорема в этом случае будет доказана. Заметим, что

$$
\beta_{B}\left(\Delta_{n}\right)=\beta_{B}\left(\Delta_{n_{0}}\right) \cdot \prod_{m_{k} \leqslant n} \frac{1}{2}=\prod_{m_{k} \leqslant n} \frac{1}{2} \leqslant \frac{\Psi_{1}\left(\Delta_{n}\right)}{\Psi_{1}\left(\Delta_{n_{0}}\right)}
$$

(мы использовали условие (12)). Так как $S_{n}(x)=o\left(n^{1-\alpha}\right)$, в силу утверждения 3 $\Psi\left(\Delta_{n}\right)=o\left(\Delta_{n}^{\alpha}\right)$, и при $\alpha<1$

$$
\Psi_{1}\left(\Delta_{n}\right)=\Psi\left(\Delta_{n}\right)-\varepsilon\left|\Delta_{n}\right|=o\left(\left|\Delta_{n}\right|^{\alpha}\right)
$$

Отсюда следует, что $\beta_{B}\left(\Delta_{n}\right)=o\left(\left|\Delta_{n}\right|^{\alpha}\right)$, т.е. по определению индекса точки ind ${ }_{B} x \geqslant$ $\alpha$, что и требовалось доказать. 
2. Последовательность $\left\{\Delta_{n}\right\}$ стягивается к точке $x \in \mathbb{R}$ и существует положительное число $\varepsilon_{0}$ такое, что $\underline{\lim }_{k \rightarrow \infty} \tau_{m_{k}} \geqslant \varepsilon_{0}$. Тогда при больших $k$

$$
\begin{aligned}
\frac{\Psi\left(\Delta_{m_{k}}\right)-\Psi\left(\Delta_{m_{k}-1}\right) / 2}{\left|\Delta_{m_{k}}\right|} & =\frac{\Psi_{1}\left(\Delta_{m_{k}}\right)-\Psi_{1}\left(\Delta_{m_{k}-1}\right) / 2}{\left|\Delta_{m_{k}}\right|}=\frac{2 \tau_{m_{k}} \cdot \Psi_{1}\left(\Delta_{m_{k}-1}\right)}{\left|\Delta_{m_{k}}\right|} \\
& \geqslant \varepsilon_{0} \cdot \Psi_{1}\left(\Delta_{n_{0}}\right) \cdot \prod_{l \leqslant k-1}\left(\frac{1+\tau_{m_{l}}}{2}\right) \frac{1}{2^{m_{k}}} \\
& \geqslant \varepsilon_{0} \cdot \frac{\Psi_{1}\left(\Delta_{n_{0}}\right) \cdot \frac{1}{2^{m_{k}-n_{0}}}}{\frac{1}{2^{m_{k}}}}=\varepsilon_{0} \cdot 2^{n_{0}} \Psi_{1}\left(\Delta_{n_{O}}\right)>0
\end{aligned}
$$

(в выкладке использовалась формула (12)). Значит, в точке $x$ ряд Хаара не сходится $\mathrm{k}$ нулю и $x \in A$. Так же, как и в варианте 1 , построим совершенное множество $B=\{x\} \cup$ $\bigcup_{k=1}^{\infty} L\left(\overline{\Delta_{m_{k}-1} \backslash \Delta_{m_{k}}}\right) \subset A$. Покажем, что ind ${ }_{B} x \geqslant \alpha$, завершив так же, как и вьше, доказательство теоремы.

В силу утверждения $4\left(\Psi\left(\Delta_{n}\right)-\Psi\left(\Delta_{n-1}\right) / 2\right) /\left|\Delta_{n}\right|^{\alpha}=o(1)$. Преобразуем выражение в числителе: $\Psi\left(\Delta_{n}\right)-\Psi\left(\Delta_{n-1}\right) / 2=\Psi_{1}\left(\Delta_{n}\right)-\Psi_{1}\left(\Delta_{n-1} / 2\right)$. Это больше или равно $\Psi_{1}\left(\Delta_{n-1}\right) / 2$, если $n \neq m_{k}$ ни для какого $k$, и равно $\tau_{m_{k}} \Psi_{1}\left(\Delta_{n-1}\right) / 2$, если $n=m_{k}$. Значит, либо $\Psi_{1}\left(\Delta_{n-1}\right) / 2=o\left(\left|\Delta_{n}\right|^{\alpha}\right)$ в первом случае, либо $\tau_{m_{k}} \Psi_{1}\left(\Delta_{n-1}\right) / 2=o\left(\left|\Delta_{n}\right|^{\alpha}\right)$ во втором случае. Но так как $\underline{\lim }_{k \rightarrow \infty} \tau_{m_{k}} \geqslant \varepsilon_{0}$, при любом варианте

$$
\Psi_{1}\left(\Delta_{n-1}\right)=o\left(\left|\Delta_{n}\right|^{\alpha}\right)
$$

Но раз $\beta_{B}\left(\Delta_{n}\right) \leqslant \Psi_{1}\left(\Delta_{n}\right) / \Psi_{1}\left(\Delta_{n_{0}}\right)$ (см. выкладку в первом варианте), то

$$
\beta_{B}\left(\Delta_{n}\right)=o\left(\left|\Delta_{n}\right|^{\alpha}\right) \text {. }
$$

Значит, $\operatorname{ind}_{B} x \geqslant \alpha$, что и завершает доказательство теоремы в этом случае.

3. Последовательность $\left\{\Delta_{n}\right\}$ стягивается к точке $x \in \mathbb{R} и \underline{\lim }_{k \rightarrow \infty} \tau_{m_{k}}=0$. Выберем последовательность $A_{s}$ такую, что

$$
A_{s}<1, \quad A_{s} \rightarrow 1 \quad \text { и } \quad \prod_{s=0}^{\infty} A_{s}=D>0 .
$$

Так как $x \in \mathbb{R}$, то найдется такое натуральное $N_{1}$, что все двоичные интервалы $\Delta_{n}$ при $n \geqslant N_{1}$ имеют один и тот же тип. Найдем $\tau_{m_{k_{0}}}$ такое, что $m_{k_{0}}>N_{1}$ и $1-\tau_{m_{k_{0}}}>$ $A_{0}$. Построим новую вложенную последовательность двоичных интервалов $\Delta_{n}^{1}$ и новую числовую последовательность $\tau_{m_{k}}^{1}$.

Пусть $\Delta_{n}^{1}=\Delta_{n}$, если $n_{0} \leqslant n<m_{k_{0}}, \tau_{m_{i}}^{1}=\tau_{m_{i}}$ при $i<k_{0}$ и, кроме того, $\Delta_{m_{k_{0}}}^{1}=$ $\overline{\Delta_{m_{k_{0}}-1} \backslash \Delta_{m_{k_{0}}}}$ и $\tau_{m_{k_{0}}}^{1}=0$. Заметим, что в силу условия (12) и в силу выбора $k_{0}$ имеем неравенство

$$
\Psi_{1}\left(\Delta_{m_{k_{0}}}^{1}\right) \geqslant \Psi_{1}\left(\Delta_{n_{0}}\right) \cdot A_{0} \cdot \prod_{i<k_{0}}\left(\frac{1+\tau_{m_{i}}}{2}\right) .
$$

Кроме того, тип $\Delta_{m_{k_{0}}}^{1}$ не совпадает с типом $\Delta_{m_{k_{0}}-1}$ (заменив двоичный интервал $\Delta_{m_{k_{0}}}$ на интервал $\Delta_{m_{k_{0}}}^{1}$, мы поменяли тип). Далее, имея набор $\Delta_{n}^{1}$ при $n_{0} \leqslant n \leqslant m_{k_{0}}$ и набор $\tau_{m_{1}}^{1}, \ldots, \tau_{m_{k_{0}}}^{1}$, будем строить последовательности $\Delta_{n}^{1}$ при $n>m_{k_{0}}$ и $\tau_{m_{k}}$ при $k>k_{0}$ 
процедурой, описанной после леммы. При этом мы опять попадем либо в один из вариантов 1 или 2 , доказав тем самым теорему, либо снова попадем в вариант 3 и тогда снова совершим процедуру, описанную в этом варианте. В итоге мы либо за конечное число шагов попадем в один из вариантов 1 или 2, тем самым доказав теорему, либо все время будем попадать в вариант 3 . В последнем случае мы построим новые последовательности $\Delta_{n}^{\infty}$ и $\tau_{m_{k}}^{\infty}$ со следующими свойствами.

1) $\Delta_{n_{0}}^{\infty}=\Delta_{n_{0}}$.

2) Существуют такие номера $m_{k_{0}}<m_{k_{1}}<\cdots<m_{k_{n}}<\cdots$, что $\Psi_{1}\left(\Delta_{m_{k_{i}}}^{\infty}\right) \geqslant$ $A_{i} \cdot \Psi_{1}\left(\Delta_{m_{k_{i}-1}}^{\infty}\right) / 2$; если $n \neq m_{k}$ ни для какого $k$, то $\Psi_{1}\left(\Delta_{n}^{\infty}\right) \geqslant \Psi_{1}\left(\Delta_{n-1}^{\infty}\right)$; если же $n=m_{k}$ для некоторого $k$, причем $k \neq k_{i}$ ни для какого $i$, то

$$
\Psi_{1}\left(\Delta_{n}^{\infty}\right)=\frac{\Psi_{1}\left(\Delta_{n-1}^{\infty}\right)}{2}\left(1+\tau_{n_{k}}\right), \quad \text { a } \quad \Psi_{1}\left(\overline{\Delta_{n-1}^{\infty} \backslash \Delta_{n}^{\infty}}\right)=\frac{\Psi_{1}\left(\Delta_{n-1}^{\infty}\right)}{2}\left(1-\tau_{n_{k}}\right) .
$$

Последовательность же $\tau_{m_{k}}^{\infty}$ обладает тем свойством, что $\tau_{m_{k_{i}}}^{\infty}=0$, a $\tau_{m_{k}}^{\infty} \geqslant 0$ для любого $k$.

3) Тип двоичного интервала $\tau_{m_{k_{i}}}^{\infty}$ не совпадает с типом $\tau_{m_{k_{i}}-1}^{\infty}$.

Другими словами, каждый кусок $\Delta_{m_{k_{i}}}^{\infty}, \ldots, \Delta_{m_{k_{i+1}-1}}^{\infty}$ построен, исходя из двоичного интервала $\Delta_{m_{k_{i}}}^{\infty}$ с помощью процедуры, описанной после леммы, а вся последовательность $\Delta_{n}^{\infty}$ состоит из таких кусков. При этом

$$
\Psi_{1}\left(\Delta_{n}^{\infty}\right) \geqslant \Psi_{1}\left(\Delta_{n}^{\infty}\right) \cdot \prod_{m_{k} \leqslant n} \frac{1+\tau_{m_{k}}^{\infty}}{2} \cdot \prod_{m_{k_{i}} \leqslant n} A_{i} \geqslant \Psi_{1}\left(\Delta_{n}^{\infty}\right) \cdot \prod_{m_{k} \leqslant n} \frac{1+\tau_{m_{k}}^{\infty}}{2} \cdot D .
$$

А так как из-за вьполнения свойства 3 ) последовательность $\Delta_{n}^{\infty}$ стягивается к точке $x \in \mathbb{I}$, в силу последних неравенств

$$
\frac{\Psi_{1}\left(\Delta_{n}^{\infty}\right)}{\left|\Delta_{n}^{\infty}\right|} \geqslant \frac{D \cdot \Psi_{1}\left(\Delta_{n_{0}}\right) \cdot \frac{1}{2^{n-n_{0}}}}{2^{n}}=D \cdot \Psi_{1}\left(\Delta_{n_{0}}\right) \cdot 2^{n_{0}}>0
$$

Тем самым ряд Хаара в точке $x$ расходится. Уже применявшимся способом строим совершенные множества $L\left(\overline{\left.\Delta_{m_{k}-1}^{\infty} \backslash \Delta_{m_{k}}^{\infty}\right)} \subset A\right.$ и строим множество

$$
B=\{x\} \cup \bigcup_{k=1}^{+\infty} L\left(\overline{\left.\Delta_{m_{k}-1}^{\infty} \backslash \Delta_{m_{k}}^{\infty}\right)}\right.
$$

которое также будет совершенньм и принадлежашим $A$. Заметим, что в силу неравенства (13)

$$
\beta_{B}\left(\Delta_{n}^{\infty}\right)=\beta_{B}\left(\Delta_{n_{0}}^{\infty}\right) \prod_{m_{k} \leqslant n} \frac{1}{2} \leqslant \frac{1}{D} \frac{\Psi_{1}\left(\Delta_{n}^{\infty}\right)}{\Psi_{1}\left(\Delta_{n_{0}}^{\infty}\right)}
$$

Кроме того, в силу условия $S_{n}(x)=o\left(n^{1-\alpha}\right)$ и утверждения 3 имеют место равенства

$$
\Psi_{1}\left(\Delta_{n}^{\infty}\right)=\Psi\left(\Delta_{n}^{\infty}\right)-\varepsilon\left|\Delta_{n}^{\infty}\right|=o\left(\left|\Delta_{n}^{\infty}\right|^{\alpha}\right) .
$$

Таким образом, получаем, что $\beta_{B}\left(\Delta_{n}^{\infty}\right)=o\left(\left|\Delta_{n}^{\infty}\right|^{\alpha}\right)$, т.е. $\operatorname{ind}_{B} x \geqslant \alpha$, а значит ind $B \geqslant$ $\alpha$. Замечая тот факт, что $B \subset A$; этим мы завершаем доказательство теоремы.

Теорема 1 представляет собой необходимое условие того, что данное множество является М-множеством для класса рядов (1) с условием (3). Прямым следствием теоремы 1 является следующая теорема. 
ТЕорема 2. Пусть $\alpha \in[0,1)$ и пусть множество А обладает следующим свойством: любое содержащееся в $А$ совершенное подмножество имеет верхний индекс, меньиий $\alpha$. Тогда $A$ является U-множеством для рядов Хаара с условием (3). В частности, теорема верна, если $A$ - совершенное множество $c$ $\overline{\operatorname{ind}} A<\alpha$.

При доказательстве второй части теоремы 2 нужно заметить, что если $C \subset A$, то $\operatorname{ind}_{C} x \leqslant \operatorname{ind}_{A} x$ для всех точек $x \in C$, а значит,

$$
\overline{\operatorname{ind}} C=\sup _{x \in C} \operatorname{ind}_{C} x \leqslant \sup _{x \in C} \operatorname{ind}_{A} x \leqslant \sup _{x \in A} \operatorname{ind}_{A} x=\overline{\operatorname{ind}} A .
$$

Следующая теорема указьвает на существование совершенньг множеств с заданными верхним и нижним индексами.

Теорема 3. Для любых чисел $\alpha$ и $\beta$ maких, что $0 \leqslant \alpha \leqslant \beta \leqslant 1$ существует совериенное мнохсество $B$ с ind $B=\beta$, ind $B=\alpha$.

ДокАЗАТЕльство. Для начала докажем несколько более слабое утверждение.

ЛЕмма. Для любого числа $\gamma, \gamma \in[0,1]$, существует совершенное множсество $A$ $c$ ind $A=\gamma$.

ДокАЗАТЕЛЬСТво ЛЕмМЫ. Пусть сначала $0 \leqslant \gamma<1$. Построим множество $A$ c ind $A=\gamma$. Пусть $p_{n}$ - последовательность целых неотрицательных чисел, причем: $p_{0}=0, p_{1}=1, p_{n}>\gamma n+\sqrt{n}$, начиная с некоторого $n(\gamma), p_{n} / n \rightarrow \gamma, p_{n}$ не убьвает и $\left(p_{n+1}-p_{n}\right)$ есть либо нуль, либо единища. Построим аддитивную функцию двоичного интервала $\Psi(\Delta)$. Положим $\Psi([0,1])=1$. Далее строим функцию $\Psi$ по индукции. Пусть $\Psi$ задана на двоичных интервалах ранга $n$. Тогда построим $\Psi$ на двоичных интервалах ранга $n+1$. Пусть $\Delta_{n}$ имеет ранг $n$, и $\Delta_{n}=\bigcup_{i=1}^{2} \Delta_{n+1}^{i}$, где $\left\{\Delta_{n+1}^{i}\right\}$ - пара неперекрываюшихся двоичных интервала ранга $n+1$. Функция $\Psi(\Delta)$ задана на $\Delta_{n}$; определим ее на $\Delta_{n+1}^{i}$. Если $\Psi\left(\Delta_{n}\right)=0$, то положим $\Psi\left(\Delta_{n+1}^{i}\right)=0$ для $i=1,2$. Если $\Psi\left(\Delta_{n}\right)>0$ и $p_{n+1}-p_{n}=1$, то $\Psi\left(\Delta_{n+1}^{i}\right)=\Psi\left(\Delta_{n}\right) / 2$ для любого $i=1,2$. Если же $\Psi\left(\Delta_{n}\right)>0$ и $p_{n+1}-p_{n}=0$, то положим $\Psi\left(\Delta_{n+1}^{i}\right)=\Psi\left(\Delta_{n}\right)$ для того двоичного интервала $\Delta_{n+1}^{i}$, тип которого $k$ таков, что интервал $\Delta_{n}$ имеет тип $\bar{k}$; для другого двоичного интервала $\Delta_{n+1}^{i}$ положим $\Psi\left(\Delta_{n+1}^{i}\right)=0$. Заметим, что для любого двоичного интервала $\Delta_{N}$ ранга $N \Psi\left(\Delta_{N}\right)$ равняется либо $1 / 2^{p_{N}}$, либо 0 .

Положим $A=\bigcap_{n} \bigcup_{\substack{\Psi\left(\Delta_{n}\right)>0 \\\left|\Delta_{n}\right|=1 / 2^{n}}} \Delta_{n}$. Заметим, что кромевсего прочего $A$ состоит только из двоично-иррациональных точек. Учитьвая это, легко заметить, что $\beta_{A}(\Delta) \equiv$ $\Psi(\Delta)$ (все интервалы, содержащие точки из $A$, содержат на самом деле бесконечное число точек из $A$ ). Пусть $x \in A$, а $\left\{\Delta_{n}\right\}$ - основная последовательность двоичных интервалов для $x$. Тогда

$$
\beta_{A}\left(\Delta_{n}\right)=\Psi\left(\Delta_{n}\right)=\frac{1}{2^{p_{n}}} \leqslant \frac{1}{2^{n \gamma+\sqrt{n}}}=\frac{1}{2^{n \gamma}} \cdot \frac{1}{2^{\sqrt{n}}}=\frac{1}{2^{\sqrt{n}}}\left|\Delta_{n}\right|^{\gamma}=o\left(\left|\Delta_{n}\right|^{\gamma}\right) .
$$

$\mathrm{C}$ другой стороны, в силу того, что $p_{n} / n \rightarrow \gamma$, начиная с некоторого $n$ имеет место неравенство $p_{n} / n<\gamma+\varepsilon$. Следовательно,

$$
\beta_{A}\left(\Delta_{n}\right)=\frac{1}{2^{p_{n}}} \geqslant \frac{1}{2^{n(\gamma+\varepsilon)}}=\left|\Delta_{n}\right|^{\gamma+\varepsilon},
$$


поэтому $\beta_{A}\left(\Delta_{n}\right) \neq o\left(\left|\Delta_{n}\right|^{\gamma+\varepsilon)}\right)$, а так как $\varepsilon>0$ можно выбрать любым, то ind ${ }_{A} x=\gamma$. В силу произвольности точки $x \in A$ мы имеем равенство ind $A=\gamma$.

Пусть теперь $\gamma=1$. Слегка видоизменим предыдущее доказательство, а именно, возьмем последовательность $p_{n}$ целых неотрицательных чисел такую, что $p_{0}=0, p_{1}=$ $1, p_{n} / n \rightarrow 1$ и $0 \leqslant p_{n+1}-p_{n} \leqslant 1$; кроме того, последовательность выберем такой, что $p_{n}-n \rightarrow-\infty$.

Выбрав последовательность $p_{n}$ указанным вьше способом, построим аддитивную функцию двоичного интервала $\Psi(\Delta)$, затем множество $A$, причем $\beta_{A}(\Delta)=\Psi(\Delta)$. Покажем, что множество $A$ удовлетворяет нужньм нам условиям. В силу равенства

$$
\lim _{n \rightarrow \infty} \frac{p_{n}}{n}=1
$$

имеем соотношение $p_{n}>n(1-\varepsilon)$ для некоторого $\varepsilon>0$ и $n>n(\varepsilon)$. Тогда

$$
\beta_{A}\left(\Delta_{n}\right)=\frac{1}{2^{p_{n}}}<\frac{1}{2^{n(1-\varepsilon)}}=\left|\Delta_{n}\right|^{1-\varepsilon}=o\left(\left|\Delta_{n}\right|^{1-2 \varepsilon}\right) .
$$

В силу произвольности $\varepsilon>0$ имеем: $\operatorname{ind}_{A} x \geqslant 1$, а значит, $\operatorname{ind}_{A} x=1$ для любой точки $x \in A$. Условие $p_{n}-n \rightarrow-\infty$ нужно только для того, чтобы множество $A$ состояло бы только из двоично-иррациональных точек, что мы использовали вьше. Лемма полностью доказана.

Вернемся к доказательству теоремы. Построим согласно лемме множество $A_{1} \mathrm{c}$ ind $A_{1}=\alpha$ и множество $A_{2} \mathrm{c}$ ind $A_{2}=\beta$. Тогда множество

$$
B=\left(A_{1} \cap\left[0, \frac{1}{/} 2\right]\right) \cup\left(A_{2} \cap\left[\frac{1}{2}, 1\right]\right)
$$

- непустое, имеет нижний индекс, равный $\alpha$, и верхний индекс, равньй $\beta$. Теорема полностью доказана.

Сформулируем теперь достаточное условие того, что данное множество будет являться М-множеством для рядов (1) с условием (3).

Теорема 4. Пусть $0 \leqslant \alpha<1$ и множество $A \subset[0,1]$ содержит совериенное подмножество $C$ ind $C>\alpha$. Тогда $A$ является М-множеством для рядов Хаара с условием (3).

ДокАЗАТЕЛЬСТво. Построим для множества $C$ аддитивную функцию двоичного интервала $\beta(\Delta)=\beta_{c}(\Delta)$ и возьмем однозначно соответствуюший этой функции ряд Хаapa, для которого $\beta(\Delta)=\sum_{n=1}^{\infty} \int_{\Delta} a_{n} \chi_{n}(x) d x$.

В точках множества $C \beta\left(\Delta_{n}\right) /\left|\Delta_{n}\right|^{\alpha}=o(1)$ из-за того, что ind $C>\alpha$, а значит, и ind $_{C} x>\alpha$ для любой точки $x \in C$. Если $x \in \mathbb{I}$, то последнее равенство означает в силу утверждения 3 , что $S_{N}(x)=o\left(N^{1-\alpha}\right)$, а если $x \in \mathbb{R}$, то

$$
\frac{\beta\left(\Delta_{n}^{1}\right)+\beta\left(\Delta_{n}^{2}\right)}{\left|\Delta_{n}^{1}\right|^{\alpha}}=o(1), \quad \frac{\beta\left(\Delta_{n}^{i}\right)-\frac{1}{2} \beta\left(\Delta_{n}^{i}\right)}{\left|\Delta_{n}^{i}\right|^{\alpha}}=o(1)
$$

что тоже означает, что $S_{N}(x)=o\left(N^{1-\alpha}\right)$. Таким образом, соответствующий рядХаара удовлетворяет условию (3). 
Остается проверить, что $S_{N}(x) \rightarrow 0$ вне множества $C$, а значит, и вне множества $A$. Тем самым, будет доказано, что $A$ - М-множество для рядов (1) с условием (3). Но если $x \notin C$, то для любой последовательности $\left\{\Delta_{n}\right\}$, основной для точки $x$, вьполняется равенство $\beta_{C}\left(\Delta_{n}\right)=0$ при $n \geqslant n_{0}(x)$. Тогда если $x \in \mathbb{I}$, то $S_{2^{n}}(x)=\beta_{C}\left(\Delta_{n}\right) /\left|\Delta_{n}\right|=0$ для любого $n \geqslant n_{0}(x)$, т.е. $S_{N}(x) \rightarrow 0$. Если же $x \in \mathbb{R}$, то обозначим за $\Delta_{n}^{i}, i=1,2$, $i$-ю основную для точки $x$ последовательность двоичных интервалов, а за $n_{0}^{i}(x)$ - тот номер, начиная с которого $\beta\left(\Delta_{n}^{i}\right)=0$. Тогда при $n \geqslant \max \left\{n_{0}^{1}(x), n_{0}^{2}(x)\right\}$ имеют место равенства

$$
\frac{\beta\left(\Delta_{n}^{1}\right)+\beta\left(\Delta_{n}^{2}\right)}{2\left|\Delta_{n}^{1}\right|}=0, \quad \frac{\beta\left(\Delta_{n}^{i}\right)-\frac{1}{2} \beta\left(\Delta_{n}^{i}\right)}{\left|\Delta_{n}^{i}\right|}=0 .
$$

Отсюда получаем в силу утверждения 2 , что если $x \notin C$ и $x \in \mathbb{R}$, то $S_{N}(x) \rightarrow 0$. Теорема полностью доказана.

ЗАмЕчАнИЕ. Отметим, что если в теореме $3 \alpha=0$, то знак ">" можно заменить на " $\geqslant$ ". Действительно, если $\alpha=0$, то $\beta_{C}=o(1)=o\left(\left|\Delta_{n}\right|^{0}\right)$ и применимы все остальные рассуждения.

Теоремы 2-4 обобщают результаты Г. М. Мушегяна, полученные им в работе [5]. В теореме 2 этой работы доказывается наличие совершенных U-множеств при условии, что обший член рядов (1) ограничен. В этом случае $S_{N}(x) \leqslant 2 n C$, если $2^{n-2}<N \leqslant$ $2^{n-1}$, т.е. $S_{N}(x)=o(\log N)$, а теоремы 2 и 3 нашей работы указывают на наличие совершенных U-множеств при более слабых условиях. Более того, теорема 3 дает способ построения таких множеств. Уже упоминавшаяся же теорема 1 из работы [5] содержится в теореме 2 и в замечании к теореме 4 нашей работы.

Кроме того, теорема 2 говорит о существовании при $0<p<2 G(p)$ U-множеств, введенных У. Уэйдом в работе [7]. Так в этой работе называются U-множества для рядов Хаара с условием $a_{n}=o\left(n^{(p-1) / 2}\right)$. Несложно показать, что это условие при $p>0$ эквивалентно условию $S_{n}(x)=o\left(n^{1-\alpha}\right)$, где $o$-равномерное по $x \in[0,1]$, а $\alpha=1-p / 2$, т.е. это условие несколько сильнее соответствующего условия (3).

\section{СПИСОК ЦИТИРОВАННОЙ ЛИТЕРАТУРЫ}

[1] Петровская М.Б. Некоторые теоремы единственности для рядов по системе Хаара // Вестн. Моск. ун-та. Сер. 1. Матем. 1964. № 5. С. 15-28.

[2] Арутюнян Ф.Г., Талалян А. А. О единственности рядов по системам Хаара и Уолша // Изв. АН СССР. Сер. матем. 1964. Т. 28. С. 1391-1408.

[3] Скворцов В. А. Теорема типа Кантора для системы Хаара // Вестн. Моск. ун-та. Сер. 1. Матем. 1964. № 5. С. 3-6.

[4] McLaughlin J., Price J. J. Comparision of Haar series with gaps with trigonometric series // Pasific J. Math. 1969. V. 28. № 3. P. 623-627.

[5] Мушегян Г. М. О множествах единственности для системы Хаара // Изв. АН АрмССР. Сер. матем. 1967. Т. 2. №6. С. 350-361.

[6] Скворцов В.А.Дифференцирование относительно сетей и ряды Хаара // Матем. сб. 1968. T. 4. № 1. C. 33-40.

[7] Wade W. R. Sets of uniqueness for Haar series // Acta Math. Academiae Scientiarum Hungaricae. 1977. V. 30. № 3-4. P. 265-281.

Вологодская государственная молочно-хозяйственная академия им. Н. В. Верещагина 